\title{
Heating of Biological Tissues by Gold Nano Particles: Effects of Particle Size and Distribution
}

\author{
Badia Fasla, Assia Rachida Senoudi, Abdelhak Boussaid, Mustapha Benmouna, Reda Benmouna
}

Macromolecular Research Laboratory, Department of Physics, Faculty of Sciences University Abou Bekr Belkaid, Tlemcen, Algeria Email: redabenmouna@yahoo.com

Received November $4^{\text {th }}, 2010$; revised November $23^{\text {rd }}, 2010$; accepted December $1^{\text {st }}, 2010$.

\begin{abstract}
This paper deals with hyper thermal therapy of tumors in biological tissues using dispersions of gold nano spheres. These spheres are heated with a laser beam in the near infrared range based on surface plasmon resonance phenomenon. The single sphere problem gives a surface temperature rise following a universal form with a characteristic time $\tau$ proportional to the sphere surface and inverse diffusivity of the surrounding medium. The temperature front is found to reach a finite range when traveling into the surrounding medium with a certain time delay. The many particles problem is treated as a convolution product of the sphere density distribution function and the particle temperature profile. Different space distribution functions of nano particles are considered. A uniform sphere distribution provides a good coverage of medium heating while a Gaussian distribution predicts an important drop of temperature when approaching the borders of the treated region. Lorentzian distribution was also considered for comparison. An effort is made to highlight the impacts of the obtained results in developing strategies for hyper thermal therapy in a joint effort with the medical team.
\end{abstract}

Keywords: Hyper Thermal Therapy, Gold Nano Spheres, Laser, Heat Transfer

\section{Introduction}

Thermal therapy has been used for a long time to treat a variety of tumors. Many years ago, Egyptians used heated papyrus to treat breast cancer and others infections [1]. Nowadays, laser thermal therapy is becoming one of the major tools of treating a variety of diseases [2]. The method is based on the interaction of radiation with soft matter knowing that biological tissues contain radiation absorbing agents such as water, proteins, hemoglobin and melanin with different absorption spectra. Laser therapy aims at eliminating cancerous tissues by heating as an alternative to conventional surgical treatments with the advantages of being non invasive, simple allowing a rapid recovery [3-6]. It is however a complex process involving a combination of distinct phenomena that require mathematical models to improve our understanding of the system behavior at different stages of the process. Energy delivered by radiation to tissue depends on parameters like laser power, beam diameter and wavelength. Thermal effects depend on the absorption cross section of tissues, their optical and thermo-physical parameters as well as on biological denaturation. The extend of tissue damage can be evaluated knowing the time and space temperature dependence. For example, vasodilatation phenomenon and endothelial damages appear at $45^{\circ} \mathrm{C}$ leading to cell's death within seconds. Damages speed up fast upon further heating.

The present paper deals with laser therapy using a dispersion of gold nano spheres. In order to minimize damages to healthy tissues surrounding the target, one needs to design strategies that include a good distribution of heating sources and a precise resolution of the heat transfer problem in space and time under practical conditions of applications. [7-9] Thermal ablation resulting from heated gold nano spheres is due to surface plasmon resonance phenomenon where gold absorbs radiation in the near infrared and release heat [10-13]. This resonance generates photon capture several orders of magnitude larger than standard photo thermal dyes [14,15]. Moreover, biological tissues are characterized by a relatively good transparency in the near infrared providing the possibility to reach deep regions via fiber optics for remote tumors deep inside the body. A model is presented here for solving the time and space heat transfer problem in spherical coordinates. The single particle problem is examined first to obtain the rate of temperature rise at the sphere surface and immediate surroundings. The case of 
a large number of nano spheres is then examined based on selected particle distributions.

\section{The Single Particle Problem}

Conducting electrons of gold nano spheres exhibit a resonance with the incident electromagnetic field and oscillate producing heat. Resonance at a specific frequency of the applied field is called plasmon resonance. Optimization of process efficiency and enhancement of thermal conversion require an adequate choice of the laser wavelength consistent with the particle shape and size.[13,14] In this section heating by a single gold sphere of radius $a$ is considered. The sphere is exposed to a laser beam of wavelength $\lambda$, intensity $I_{0}\left(\mathrm{~W} / \mathrm{m}^{2}\right)$ and assumed to generate a power proportional to the absorption cross section $\sigma_{\text {abs, }} P_{a b s}=I_{0} \sigma_{a b s}(\mathrm{~W})$. This cross section is different from the geometric expression (i.e., $4 \pi a^{2}$ ) and can be evaluated depending on dielectric properties of the medium. When an electromagnetic radiation of wavelength $\lambda$ impinges into the sphere, its intensity splits into absorption and scattering. The absorption power which is more relevant for our purpose depends on the cross section $\sigma_{a b s}$. According to the electro-dynamic theory of dielectric media, absorption cross section is related to the polarizability of the sphere $\alpha[16,17]$ by $\sigma_{a b s}=\left[(2 \pi / \lambda) / \varepsilon^{1 / 2}\right] \operatorname{Im} \alpha$, and the polarizability can be deduced from the Clausius-Mossotti $[18,19]$ relationship as $\alpha=4 \pi a^{3}(\varepsilon-$ $\left.\varepsilon_{m}\right) /\left(\varepsilon+2 \varepsilon_{m}\right)$ where $\varepsilon$ is the sphere dielectric permittivity and $\varepsilon_{m}$ that of the biological medium in which it is embedded (i.e., the physiological liquid for example). The permittivity $\varepsilon$ is a complex quantity with real and imaginary parts that can be evaluated in terms of gold parameters and surrounding medium using Lorentz-Drude models. [20,21] It is not our attention to dwell more on this question which is nevertheless important to complete our understanding of the problem. A more detailed investigation along these lines is under progress and we hope to report on some results in the near future essentially for confronting the conversion efficiencies with those of other metals such as iron, nickel, silver or hybrid spheres containing polymers [22].

The problem now consists of solving the following sets of equations

$$
\begin{gathered}
\lambda_{g}^{-1} \frac{\partial T_{g}}{\partial t}-\frac{1}{r^{2}} \frac{\partial}{\partial r}\left(r^{2} \frac{\partial T_{g}}{\partial r}\right)=\frac{P_{a b s}}{\kappa_{g}} \text { for } r<a \\
\lambda^{-1} \frac{\partial T}{\partial t}-\frac{1}{r^{2}} \frac{\partial}{\partial r}\left(r^{2} \frac{\partial T}{\partial r}\right)=0 \quad \text { for } r \geq a
\end{gathered}
$$

Subscript $g$ refers to gold while $T$ is the temperature in the surrounding medium; $\lambda$ 's and $\kappa^{\prime}$ s are thermal diffusivities $\left(\mathrm{m}^{2} / \mathrm{s}\right)$ and conductivities $\left(\mathrm{W} \cdot \mathrm{m}^{-1} \cdot \mathrm{K}^{-1}\right)$, respectively. The focus is made on the resolution of Equation (2) since $T(r, t)$ is the relevant temperature. In addition, the solution gives access to $T_{\mathrm{s}}$ at the sphere surface which is sufficient to characterize the heating source. The solution must satisfy the following initial and boundary conditions:

$$
\begin{gathered}
\text { Initial condition } T(r, t=0)=T_{0} \\
\text { Boundary conditions } T(r=\infty, t)=T_{0} \\
-\left.\kappa \frac{\partial T}{\partial r}\right|_{r=a}=\frac{P_{a b s}}{4 \pi a^{2}}
\end{gathered}
$$

$T_{0}$ is the initial temperature prior to laser exposure and remains that of healthy tissue away from the infected region. Equation 5 expresses the heat flux from the sphere.

To solve equation 2 subject to the indicated initial and boundary conditions, we first make a change of variable from $T(r, t)$ to $u(r, t)=r\left[T(r, t)-T_{0}\right]$ then Laplace transform the resulting equation. Resolution of the equation in $u(r, t)$ is straight forward. The final step is to go back to time domain and noting that the inverse Laplace transform of $a \exp (-k \sqrt{s}) / s(a+\sqrt{s}) \quad(k>0)$ is tabulated in [23] as $\operatorname{erfc}(k / 2 \sqrt{t})-\exp \left(a k+a^{2} t\right) \operatorname{erfc}(a \sqrt{t}+k / 2 \sqrt{t})$.

The result is

$$
\begin{aligned}
& \Delta T_{1}(r, t)=T(r, t)-T_{0} \\
& =\Delta T_{1 \max } \frac{a}{r}\left\{\begin{array}{l}
\operatorname{erfc}\left(\frac{r-a}{2 a \sqrt{t \tau^{-1}}}\right) \\
-\exp \left(\frac{r-a}{a}+t \tau^{-1}\right) \operatorname{erfc}\left(\frac{r-a}{2 a \sqrt{t \tau^{-1}}}+\sqrt{t \tau^{-1}}\right)
\end{array}\right\}
\end{aligned}
$$

where

$$
\Delta T_{1 \max } \equiv \frac{P_{a b s}}{4 \pi a \kappa} ; \tau \equiv \frac{a^{2}}{\lambda} ; \lambda \equiv \frac{\kappa}{\rho c_{p}}
$$

and $c_{P}$ represent the sphere density and specific heat, respectively; erfc is the complementary error function

$$
\operatorname{erfc} X=\frac{2}{\sqrt{\pi}} \int_{X}^{\infty} d t e^{-t^{2}}=1-\operatorname{erf} X
$$

The subscript 1 is used to distinguish single particle from many particle temperature fields to be discussed in the next section; $\tau$ is the characteristic time of sphere photo heating. The procedure used to solve this problem is similar to that reported in [24] where the coupled equations for $r<a$ and $r>a$ were solved. Note that Equation (6) is similar to the result of Kablinski et al. [10].

The characteristic time is proportional to the sphere surface and inverse thermal diffusivity of the surrounding medium meaning that it lasts longer to heat bigger spheres embedded in poorly diffusing media. Moreover, $\Delta T_{1 \max }=$ $P_{a b s} / 4 \pi a \kappa$ is proportional to $P_{a b s}$ and inversely proportional to sphere's radius and tissue's thermal conductivity. 
$P_{a b s}$ was discussed above in terms of laser intensity $I_{0}$ and wavelength $\lambda$ as well as the sphere characteristics. It should be kept in mind that absorption decreases as the sphere radius increases because of enhanced scattering. This may lead to substantial reduction in $\Delta T_{1 \max }$ and concomitant inefficacy of thermal treatment.

The sphere surface temperature is obtained by letting $r$ $=a$ in Equation (6):

$$
\frac{\Delta T_{s}(t)}{\Delta T_{1 \max }}=1-\exp \left(\frac{t}{\tau}\right) \operatorname{erfc} \sqrt{\frac{t}{\tau}}
$$

Temperature inside the sphere is irrelevant for our purpose since what really matters is $\Delta T_{1}(r, t)$ or eventually its surface limit $\Delta T_{\mathrm{s}}(t)$. The time rise of temperature at the immediate vicinity of the source is inversely proportional to thermal diffusivity $\lambda$ It is rather low depending on thermo-physical properties of tissue. Furthermore, $\tau$ is proportional to the sphere surface meaning that local tissue heating is practically instantaneous. The amplitude of heating represented by the factor $\Delta T_{1 \max }=P_{a b s} / 4 \pi a \kappa$ implies that the increase of surface temperature can be modulated by the absorbed power for a given tissue of conductivity $\kappa$. Temperature increases linearly with the absorbed power but this increase is tempered for highly conducting tissues which is expected. With regards to the sphere radius, from the points of view of fast time rise and higher local temperatures, it would be suitable to use smaller heating sources. Note that if the sphere radius exceeds $40 \mathrm{~nm}$, surface plasmon resonance phenomenon shifts to near infrared and the laser device should be adapted accordingly [25]. These findings represent qualitative guidelines in the choice of optimal conditions of hyper thermal laser treatment. To be more specific, we give in Figure 1 plots of $\Delta T_{1}(r, t) / \Delta T_{1 \max }$ against $t / \tau$ in the immediate vicinity of the sphere. The upper curve reproduces the surface temperature $\Delta T_{S}(\mathrm{t}) / \Delta T_{1 \max }$ which is a universal plot in terms of $t / \tau$ while the others give the temperature as one goes away from the heating source.

Local tissue heating is increasingly delayed and dumped with distance $r$. This is illustrated in the amplification of initial stages shown in the figure inset. Heating at a distance $r=3 a$ starts with a delay of $34 \mathrm{~ns}$ while temperature reached only $0.65 \Delta T_{1 \max }$ at $t=10 \tau$. One would have to wait $100 \tau=10^{4} \mathrm{~ns}$ to attain $0.94 \Delta T_{1 \mathrm{ma}}$.

Figure 2 exhibits movements of the temperature front from the source to the surrounding medium. At $t=\tau$, the temperature front drops from $\Delta T_{S} / \Delta T_{1 \max }=0.55$ to zero at point $r=5 a$. If one waits long enough (say $t=40 \tau$ ), the temperature goes from $100 \%$ at the surface to near zero at $r=20 a$. This means that heating by a single nano particle never goes beyond a distance $r=20 a$ inside the biological medium which is clearly insufficient. Therefore, many particles' heating is necessary to envisage a reasonable

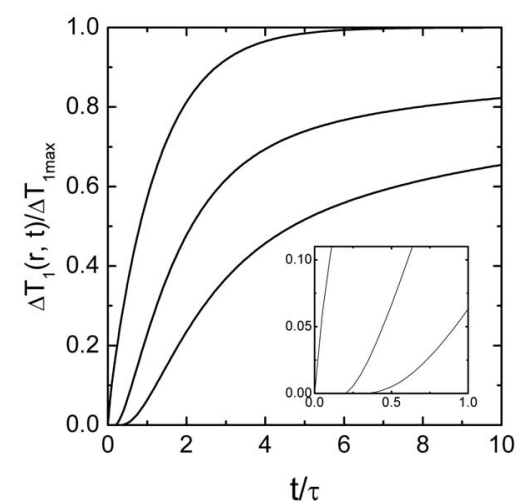

Figure 1. Single nano sphere heating $\Delta T_{1}(r, t) / \Delta T_{1 \max }$ versus $t / \tau$ as given by Equation (6) at different locations in the biological tissue: in the descending order $r / a=1,2,3$. The case $\mathbf{r} / \mathbf{a}=1$ gives the surface heating $\Delta T_{S}(t) / \Delta T_{1 \max }$ consistent with Eq.(16). Inset shows an amplification at short times to illustrate heating delays.

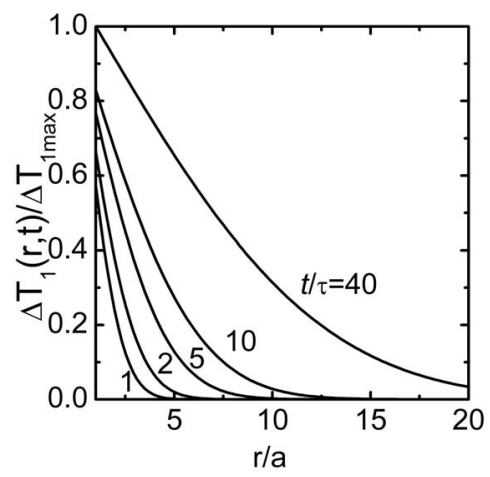

Figure 2. Single nano sphere heating $\Delta T_{1}(r, t) / \Delta T_{1 \max }$ versus $r / a$ at different times (see Eq.6). In the descending order $t / \tau=$ 40,10,5,2,1.

treatment.

Although obvious, this result should be nevertheless useful for designing an efficient thermal therapy strategy of tumor tissue that relies on collective effects of many such sources.

It describes the contribution of a single source to the local heating of the centimeter sized target material. Ways to extend this treatment to the entire tissue are discussed below based on a high number of sources properly distributed throughout the target.

\section{The Many Particles Heating Problem}

One of the main objectives in using gold nano spheres steams from the need to develop methods that lead to elimination of infected cells preserving healthy ones. The targeting approach works for diagnosis and therapy strategies. Search for an appropriate distribution of nano spheres throughout the infected region is part of this endeavor. The nano particles should become operational 
under remote activation signals with the capacity to respond to external commands in a predictable way. Intense research efforts are currently focusing on the development of novel technologies using nano metallic, organic or hybrid sources synthesized in a way to be responsive to remote electric, magnetic or electromagnetic fields. In our case, these particles must fulfill in addition to the above requirements other conditions that are related with their biocompatibility with the least toxicity to the human body.

\subsection{Uniform Particles Distribution}

Figure 3 shows a schematic representation of a uniform distribution of nano spheres throughout a centimeter sized target supposed to be spherical. The first panel describes the coordinates system while panel $b$ shows projection of the sphere onto the $\mathrm{x}-\mathrm{y}$ plane. The $\mathrm{z}$-axis gives the temperature distribution and the amplification describes the local heating due to a single particle.

As a typical example, if one considers the density of nano spheres suggested in references [4,10], namely $10^{15}$ particles $/ \mathrm{m}^{3}$, then a spherical tumor of radius $1 \mathrm{~mm}$ would contain more than 4 millions spheres, which is high number. Assuming that the laser intensity is $40 \mathrm{~W} / \mathrm{cm}^{2}$ and approximating the sphere absorption cross section with the geometric one, the power absorbed by a sphere of radius $40 \mathrm{~nm}$ would be about $10 \mathrm{nW}$. A typical conductivity of a biological tissue such as prostate, kidney or liver is in the order of $0.5 \mathrm{~m}^{-1} \mathrm{~K}^{-1}$ [26]. This example yields a single particle heating contribution $\Delta T_{1 m \text { ax }}=0.04 \mathrm{~K}$ which is of course too low for our purpose. The maximum rise of temperature in the whole target reads:

$$
\Delta T_{\max }=N \frac{a}{R} \Delta T_{1 \max }=\phi \frac{\sigma_{\mathrm{R}}}{\sigma_{\mathrm{a}}} \Delta T_{1 \max }
$$

where volume fraction $\phi$ and surface fractions $\sigma$ s are defined as:

$$
\phi=\frac{N V_{a}}{V_{R}}=\frac{N a^{3}}{R^{3}} ; \frac{\sigma_{a}}{\sigma_{R}}=\frac{a^{2}}{R^{2}}
$$

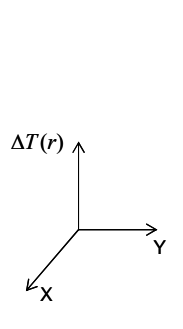

a)

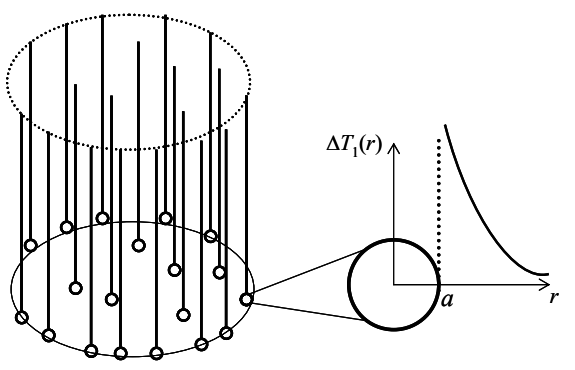

b) c)
Figure 3. Schematic representation of temperature profiles for a uniform nano spheres distribution: (a) The coordinates system; (b) Temperature and nano spheres distributions; (c) Amplification of the local heating due to a single nano sphere.
The surface ratio appearing in Equation (10) means that only gold particles respond to the laser beam. These considerations are of course approximate but have the merit of giving rough estimate of different quantities involved in the therapy process. For example, one finds that the maximum temperature rise in the tumor region is in the order of $\Delta T_{\max }=10 \mathrm{~K}$ which is sufficient for therapeutic purposes.

Heating biological tissues at a distance $r$ from the origin is a combined effect of all spheres. For the Dirac delta function distribution, the maximum rise of temperature in the biological tissue $\Delta T_{\max }$ is proportional to the volume fraction of spheres $\phi$ but for a modulated space dependent distribution, one must take into account density variations in space and introduce an average temperature rise due to the $\mathrm{N}$ particle system as:

$$
\Delta T_{N}(r, t)=\int d^{3} r^{\prime} D_{N}\left(r^{\prime}\right) \Delta T_{1}\left(r-r^{\prime}, t\right)
$$

where $D_{N}\left(r^{\prime}\right) d^{3} r^{\prime}$ is the probability of finding a particle at a distance $r$ ' in a small volume $d^{3} r$ '. Equation 12 describes the response of the medium at point $r$ due to a source located at $r$ '. Response of the biological tissue to the $N$ particle heating system has the form of a convolution product involving the space variation of density distribution function $D_{N}(\vec{r})$ and single particle heating temperature. This problem is conveniently handled in Fourier space by writing

$$
\Delta T_{N}(q, t)=D_{N}(q) \Delta T_{1}(q, t)
$$

where $q$ is the Fourier variable conjugate of $r$ and Fourier transform is defined as

$$
D_{N}(q)=\int d^{3} r \exp (i \vec{q} \vec{r}) D_{N}(r)
$$

The result of Equation (11) means that $D_{N}(r)$ is a uniform distribution of Dirac delta function, i.e.

$$
D_{N}(r)=\sum_{j=1}^{N} \delta\left(r-r_{j}\right)=N \frac{a}{R} \delta(\vec{r})=\phi \frac{\sigma_{R}}{\sigma_{a}} \delta(\vec{r})
$$

Below, we will consider the case of a Gaussian distribution of nano spheres.

\subsection{Gaussian Distribution}

Here we assume that the nano sphere density decreases from the target center according to the Gaussian distribution represented in Figure 4.

Using a similar normalization as above, one finds:

$$
D_{N}(r)=N \frac{a}{R}\left(\frac{3}{4 \pi R^{2}}\right)^{3 / 2} \exp \left(-\frac{3 r^{2}}{2 R^{2}}\right)
$$

Fourier transforming this expression and using the following integral [23]

$$
\int_{-\infty}^{+\infty} d x x \exp \left(-p x^{2}+2 q x\right)=\frac{q}{p} \sqrt{\frac{\pi}{p}} e^{\frac{q^{2}}{p}} \quad(\mathrm{p}>0)
$$




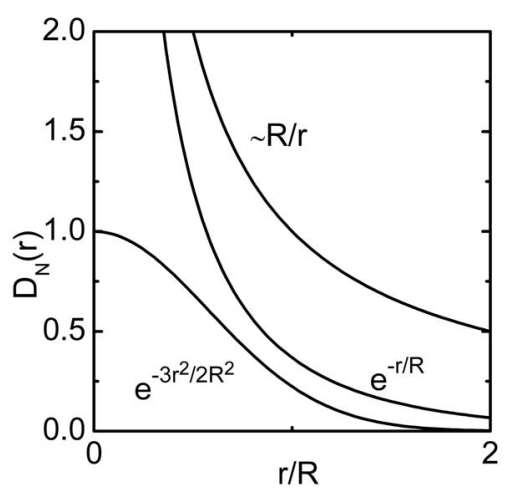

Figure 4. Nano sphere distribution function $D_{N}$ in terms of $r / R$ where $R$ is the radius of heated tissue.

One finds

$$
D_{N}(q)=\frac{N a}{R} \exp \left(-\frac{q^{2} R^{2}}{6}\right)
$$

Hence, the temperature rise in the presence of $N$ spheres becomes

$$
\Delta T_{N}(q, t)=\frac{N a}{R} e^{-\frac{q^{2} R^{2}}{6}} \Delta T_{1}(q, t)
$$

The Gaussian factor $\exp \left(-q^{2} R^{2} / 6\right)$ representing the temperature drop with $q R$ is shown in Figure 5. At $q R=1$ for example, this drop is quite substantial since.

$$
\exp \left(-\frac{1}{6}\right)=0.02778
$$

Another density distribution of nano spheres that can be considered for comparison would be

$$
D_{N}(r)=\frac{N a}{R} \frac{1}{4 \pi R^{2}} \frac{e^{-r / R}}{r}
$$

which is also represented in Figure 4. Fourier transforming yields a Lorentzian distribution in terms of $q R$

$$
D_{N}(q)=\frac{N a}{R} \frac{1}{1+q^{2} R^{2}}
$$

We mention this form because it is often encountered in soft matter physics and Brownian motion of colloidal dispersions [27,28] although its relevance to therapeutic applications could be subject to doubt. In this case, the total rise of temperature would be

$$
\Delta T_{N}(q, t)=\frac{N a}{R} \frac{1}{1+q^{2} R^{2}} \Delta T_{1}(q, t)
$$

where the factor $\left(1+q^{2} R^{2}\right)^{-1}$ represents a space modulated drop of temperature reminiscent of a Lorentzian distribution (see Figure 5). It shows that at tumor's border, the temperature drops by nearly a factor 2 which is expected since the density of nano particles is lower in this region.

Throughout these discussions, Fourier transform of

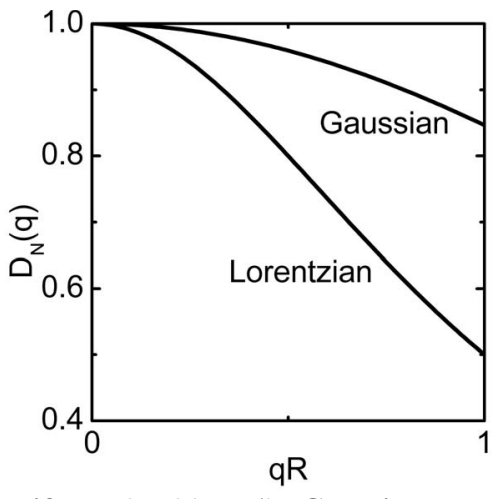

Figure 5. Uniform $\left(D_{N}(q)=1\right)$, Gaussian and Lorentzian distribution functions in Fourier space $D_{N}(q)$ versus $q R$.

$\Delta T_{1}(r, t)$ was not needed since this function could be analyzed directly in $r$-space.

\section{Discussion and Conclusion}

Hyper thermal therapy using laser heated nano sized gold spheres is analyzed by solving the heat transfer problem. It is found that the sphere temperature rises with a characteristic time that is proportional to its surface and inversely proportional to heat diffusivity of the surrounding biological medium. Heat fronts move from the particle to the medium with increasing delays as one goes away from the surface. A simple numerical example shows that heat generated by a single particle is too low to produce any noticeable effect on the surrounding tissue but the combined effects of a large number of particles is sufficient for thermal ablation. Models are used for the particle density distribution function $D(r)$ and a mean temperature rise defined as a convolution product of the distribution and the single particle temperature front. The function $D\left(r-r^{\prime}\right)$ is defined as the probability density of finding a particle at point $r$ ' knowing that there is one at point $r$. For a uniform distribution of spheres, the probability distribution would be a sum of Dirac delta functions and the problem reduces to the single particle contribution $\Delta T_{l}(r, t)$ within a constant factor. But if the density of spheres is space modulated and decreases as one goes away from the target center due to some specific long range inter particle interactions, then the probability drops substantially. We have examined the case of Gaussian and Lorentzian distributions. In the latter case, the probability distribution decays according to a long $1 / r$ tail damped exponentially as

$$
D(r)=\alpha \frac{\mathrm{e}^{-r / R}}{r}
$$

where $\alpha$ is normalization constant. A colloidal dispersion of charged particles is an example of this distribution. In the case of a Gaussian distribution, one has

$$
D(r)=\left(\frac{2}{3 \pi R^{2}}\right)^{3 / 2} e^{-\frac{r^{2}}{2 R^{2}}}
$$


an example of which would be spheres with a harmonic potential interaction. For a uniform distribution, temperature raised proportionally to $\Delta T_{1}(q)$ within a constant factor defined by the volume fraction of spheres times their fractional area. For Lorentzian and Gaussian distributions, the temperature was also proportional to $\Delta T_{1}$ modulated by a space dependent factor reminiscent of the drift in the concentration of spheres as the border of the target region was approached. Lorentzian distribution predicted a moderate drop of temperature rise compared to the Gaussian case where a much stronger exponential drop was obtained.

It is worthwhile to note that biological systems such as proteins $(10-100 \mathrm{~nm})$, cells $(10-100 \mathrm{~mm})$ and bacteria $(1-10 \mathrm{~mm})$ are characterized by a broad range of sizes. Use of nano spheres of diameter between 10 and $40 \mathrm{~nm}$ in hyper thermal therapy seems to be adequate and efficient at the proteins level. It should be kept in mind that foreign particles can react with the host medium selectively depending on a variety of conditions related with the nature of particles and biological tissue. Hydrophobic and hydrophilic particles react differently and can be covered with molecular species present in blood. They can absorb proteins and these effects should be taken into account to define the right distribution and evaluate their stability and efficiency in developing a therapy strategy [29] of tumor tissues. Magnetic nano particles such as $\mathrm{Fe}_{3} \mathrm{O}_{4}$ exhibit a magnetization that oscillates up and down under the influence of an external field and induce heating of the surrounding medium. Other elements like cobalt and nickel can also be used combined with polymers.

These results should be useful in prescribing efficient treatments of tumors in close collaboration with the medical team. It is hoped that these considerations will trigger interest in collecting precise experimental data to assess the validity of the results and the concomitant predictive models.

\section{REFERENCES}

[1] J. H. Breasted, "Edwin's Smith Chirurgical Papyrus," University of Chicago, 1930.

[2] L. Goldman, et al., Adv. Biomed. Eng. Med. Phys., Vol. 1, 1968 , pp. 317.
[3] M. Ferrari, Nat. Rev. Cancer, Vol. 5, pp. 161, 2005.

[4] I. R. Hirsh et al., Proc. Nat. Acad. Sci. USA, Vol. 100, pp. 13549, 2003.

[5] A.G. Cuenca et al., Am. Cancer Soc., Vol. 107, pp. 459 2006.

[6] O. V. Salata, J. Nanobiotech., Vol. 2, pp. 1, 2004.

[7] A. Hadj Sahraoui, et al., J. Appl. Phys., Vol. 82, pp. 12, 1997.

[8] A. Boudene, S. Khaldi, J. Appl. Polym. Sci. Vol. 89, pp. 481, 2003.

[9] X. Coqueret et al., Macromol. Theory Simul., Vol. 9, pp. 725, 2000.

[10] P. Keblinski al., J. Appl. Phys., Vol. 100, pp. 054305, 2006.

[11] C. M. Pitsillides et al. Biophys. J., Vol. 84, pp. 4023, 2003.

[12] L. E. Vlerken, M. M. Amiji, Expert Opin. Drug Deliv., Vol. 3, pp. 205, 2006.

[13] X. Huang et al., J. Adv. Res., Vol. 1, pp. 13, 2010.

[14] D. Lapotko, Nanomed., Vol. 4, pp. 253, 2009.

[15] S. Shenogin et al., J. Appl. Phys., Vol. 95, pp. 8136, 2004.

[16] G. Mie, Ann. Phys., Vol. 25, pp. 377, 1908.

[17] M. Kerker, "The Scattering of Light and Other Electromag Netic Radiations," Academic Press, New York, 1969.

[18] B.T. Draine et al., Astrophys. J., Vol. 405, pp. 685, 1993.

[19] B.T. Draine, "Light Scattering by Non-Spherical Particles: Theory, Measurements and Applications," Academic Press, San Diego, 2000.

[20] U. Kreibig, M. Vollmer, "Optical Properties of Metal Clusters,” Springer Verlag, Berlin, 1995.

[21] C.F. Bohren, D.R. Huffman, "Absorption and Scattering of Light by Small Particles," Wiley, New York, 1983.

[22] A. R. Sennoudi et al. unpublished.

[23] I.-S. Gradshteyn and I. W. Ryghik, "Tables of Integrals, Series and Products," Academic Press, New York, 1965.

[24] H. Goldenberg, C.J. Tranter, Brit. J. Appl. Phys. 296, 1952.

[25] G. Yin et al., J. Kor. Phys. Soc., Vol. 49, pp. 2108, 2006.

[26] G. Brix et al, Magn. Res. Imag., Vol. 20, pp. 65, 2002.

[27] F. Hakem et al., Ber. Bunsenges. Phys. Chem., Vol. 100, pp. 815, 1996.

[28] M. Benmouna et al., Phys. Chem. Liq., Vol. 16, pp. 235, 1987.

[29] J. M. Dobson, "Manual of Small Animal Oncology", RAS White Edit., Bsava, 1991. 